\title{
AN EXTENSION THEORY FOR A CERTAIN CLASS OF LOOPS
}

\author{
R. H. BRUCK
}

Introduction. If $E$ is a group with a normal subgroup $K$ one may form the quotient group $E / K \cong M$. Conversely, for preassigned groups $K, M$, there is the extension problem: to determine (in some sense) all groups $E$ with $K$ as normal subgroup such that $E / K \cong M$. Much progress has been made on this problem, particularly through the work of Baer $[1,2,3]^{1}$ and the cohomology theory of Eilenberg and MacLane $[1,2,3]$. The latter authors make it clear that insight is gained by relinquishing part of the associative law; specifically, by requiring that $E$ be merely a loop such that the associative law $\left(e_{1} e_{2}\right) e_{3}=e_{1}\left(e_{2} e_{3}\right)$ holds if at least one of the $e_{i}$ belongs to a distinguished subgroup of $K$. We take this to be $K$ itself. It then becomes evident that the subclass of loops $E$ consisting of the groups is not the only one of interest; one may consider, for example, the Moufang loops, in which case it seems natural to allow $M$ also to be Moufang. Thus we approach the extension problem actually studied in the paper: $M$ is a given loop, $K$ is a group (not given, but with given centre $G$ ) and $E$ is to be any loop with $K$ as a normal subloop contained in the "associator" of $E$, such that $E / K \cong M$. This problem is more typical of group theory than of loop theory but is, nevertheless, a natural and significant special topic in the theory of loops.

For the sake of brevity no examples or applications are given and references to the bibliography are kept to a minimum. The EilenbergMacLane kernels, important for constructions, have been ignored. I may signal out as new: the inverse of a (noncentral) extension ( $\$ 1$ ), the specific results on central Moufang extensions $(\$ 6)^{2}$ and the allpervading functions $F$ which generalize (even for $M$ a group) the Eilenberg-MacLane cocycles. As indicated by Theorem 8 (\$4), additional information about the functions $F$ would probably increase our knowledge of cohomology groups.

1. Extensions. A loop $M$ is a system with a multiplication such

An address delivered before the Chicago Meeting of the Society on November 25, 1949, by invitation of the Committee to Select Hour Speakers for Midwestern Sectional Meetings; received by the editors February 28, 1950.

1 Numbers in brackets refer to the bibliography at the end of the paper.

${ }^{2}$ Slightly weaker results on central Moufang extensions were obtained in 19461947 with the support of a Guggenheim Fellowship supplemented by a grant from the Wisconsin Alumni Research Foundation. (See Bull. Amer. Math. Soc. Abstract 53-1-11.) 
that: (a) in $x y=z$, any two of $x, y, z$ uniquely determine the third; (b) $M$ has a unit 1. The associator $A=A(M)$ is the subset of $M$ such that $(x y) z=x(y z)$ if at least one of $x, y, z$ is in $A$; the associator is an associative subloop (and therefore a group). A subloop $H$ of $M$ is normal in $M$ if and only if $H$ is the kernel of a homomorphism of $M$ into a loop; equivalently, $x H=H x,(x y) H=x(y H),(x H) y=x(H y)$, $(H x) y=H(x y)$ for all $x, y$ in $M$. The mapping $x \rightarrow x H$ of $M$ set up by a normal subloop $H$ is a homomorphism upon a quotient loop $M / H$. (See Bruck [1].)

If $M$ is given, we wish to study all loops $E$ such that (i) $E$ has a homomorphism $\theta$ upon $M$; (ii) the kernel $K$ of $\theta$ is a subgroup of $A(E)$. Let $G=Z(K)$ be the centre of $K$. For each $e$ in $E$ define the mapping $T(e)$ of $K$ by

$$
k e=e(k T(e)), \quad k \in K .
$$

Applying $\theta$ to both sides of (1) we see that $k T(e)$ is in $K$. And to each $k^{\prime}$ in $K$ corresponds a unique $k$ in $K$ such that $k T(e)=k^{\prime}$. Furthermore, $e\left(\left(k_{1} k_{2}\right) T(e)\right)=\left(k_{1} k_{2}\right) e=k_{1}\left(k_{2} e\right)=k_{1}\left(e \cdot k_{2} T(e)\right)=k_{1} e \cdot k_{2} T(e)$ $=\left(e \cdot k_{1} T(e)\right) \cdot k_{2} T(e)=e\left(k_{1} T(e) \cdot k_{2} T(e)\right)$. Thus $T(e)$ is an automorphism of $K:\left(k_{1} k_{2}\right) T(e)=k_{1} T(e) \cdot k_{2} T(e)$. In particular, $T(1)$ is the identity automorphism. Moreover, $\left(e_{1} e_{2}\right) \cdot k T\left(e_{1} e_{2}\right)=k\left(e_{1} e_{2}\right)=\left(k e_{1}\right) e_{2}$ $=\left(e_{1} \cdot k T\left(e_{1}\right)\right) e_{2}=e_{1}\left(k T\left(e_{1}\right) \cdot e_{2}\right)=e_{1}\left(e_{2} \cdot k T\left(e_{1}\right) T\left(e_{2}\right)\right)=\left(e_{1} e_{2}\right) \cdot k T\left(e_{1}\right) T\left(e_{2}\right)$, or $k T\left(e_{1} e_{2}\right)=k T\left(e_{1}\right) T\left(e_{2}\right)$. In other words, the mapping $e \rightarrow T(e)$ is a homomorphism of $E$ upon a group of automorphisms of $K$.

For our purposes a pair $(G, M)$ shall consist of an abelian group $G$, a loop $M$ and a single-valued product $g x$ from $G M$ to $G$ such that $g 1=g,\left(g g^{\prime}\right) x=(g x)\left(g^{\prime} x\right)$ and $(g x) y=g(x y)$ for all $g, g^{\prime}$ in $G$ and $x, y$ in $M$, where 1 is the unit of $M$. From (1), $T(e)$ is an inner automorphism if $e$ is in $K$. Thus, for arbitrary $g$ in $G=Z(K), k$ in $K, e$ in $E$, we have $g T(k e)=g T(k) T(e)=g T(e)$. However, $e^{\prime} \theta=e \theta$ if and only if $e^{\prime}=k e$ for $k$ in $K$; thus $g T(e)$ depends only on $g$ and $x=e \theta$. Hence if we set $g x=g T(e), G$ and $M$ become a pair $(G, M)$. It is a mere matter of bookkeeping (which turns out to be useful) to pursue the study in terms of a fixed pair $(G, M)$. This leads to the basic definition:

Definition 1 . Let $(G, M)$ be a pair. A $(G, M)$ extension $(E, \theta)$ consists of a loop $E$ and a homomorphism $\theta$ of $E$ upon $M$ such that (i) $K=1 \theta^{-1}$ is in $A(E)$; (ii) $Z(K)=G$; (iii) $g e=e(g x)$ for $g$ in $G, e$ in $E$, $x=e \theta$.

It will be convenient to list here other fundamental definitions concerning extensions.

Definition 2. $(E, \theta)$ is central if $1 \theta^{-1}=G$.

Definition 3. $\left(E_{1}, \theta_{1}\right)$ is equivalent to $\left(E_{2}, \theta_{2}\right)$ if there exists an iso- 
morphism $\pi$ of $E_{1}$ upon $E_{2}$ such that (i) $\theta_{1}=\pi \theta_{2}$; (ii) $g \pi=g$ for $g$ in $G$. (Notation: $E_{1} \sim E_{2}$.)

Equivalence is reflexive, symmetric, transitive; it will serve as equality. Equivalence should be contrasted with inverse equivalence:

DEFINITION 4. $\left(E_{1}, \theta_{1}\right)$ is inverse equivalent to $\left(E_{2}, \theta_{2}\right)$ if there exists an isomorphism $\pi$ of $E_{1}$ upon $E_{2}$ such that (i) $\theta_{1}=\pi \theta_{2}$; (ii) $g \pi=g^{-1}$ for $g$ in $G$. (Notation: $E_{1} \sim^{-1} E_{2}$.)

Inverse equivalence is symmetric, not always reflexive. Transitivity has three substitutes, one being: $E \sim^{-1} E_{1}, E_{1} \sim E_{2}$ imply $E \sim^{-1} E_{2}$. Therefore, since equivalence is to serve as equality, we may define the inverse $(E, \theta)^{-1}$ as any extension inverse equivalent to $(E, \theta)$. The inverse of $(E, \theta)$ may be constructed as follows. Let $u(x)$ be any normalized system of representatives of $M$ in $E$; thus $u(x) \theta=x, u(1)$ $=1$. If $K=1 \theta^{-1}$, every $e$ in $E$ has a unique representation $e=u(x) k$ with $x=e \theta, k$ in $K$; define $\pi$ by $e \pi=u(x) k^{-1}$. Define a new operation (o) on the elements of $E$ by $e o e^{\prime}=\left(e \pi \cdot e^{\prime} \pi\right) \pi$; it is easy to see that this turns $E$ into a loop $E^{-1}$. I claim that $\left(E^{-1}, \theta\right)$ is the desired inverse. Indeed, $\pi$ is an isomorphism of $E$ upon $E^{-1}$, and $g \pi=g^{-1}$ for $g$ in $G$. Also $\theta=\pi \theta$. Certainly $\theta$ is a homomorphism of $E^{-1}$ upon $M$, the kernel being the group $K \pi$ anti-isomorphic to $K$, with centre $G \pi=G$. If at least one of $e_{1}, e_{2}, e_{3}$ is in $K \pi,\left(e_{1} o e_{2}\right) o e_{3}=\left(\left(e_{1} \pi \cdot e_{2} \pi\right) \cdot e_{3} \pi\right) \pi$ $=\left(e_{1} \pi \cdot\left(e_{2} \pi \cdot e_{3} \pi\right)\right) \pi=e_{1} o\left(e_{2} o e_{3}\right)$; thus $K \pi$ is in $A\left(E^{-1}\right)$. For $g$ in $G, e$ in $E^{-1}, x=e \theta$, we have $g o e=\left(g^{-1} \cdot e \pi\right) \pi=\left(e \pi \cdot\left(g^{-1} x\right)\right) \pi=e o(g x)$. This completes the proof.

Definition 5. The product $\left(E_{1}, \theta_{1}\right) \otimes\left(E_{2}, \theta_{2}\right)=(E, \theta)$ of two extensions $\left(E_{j}, \theta_{j}\right)$ is defined as follows: (i) The elements of $E$ are the pairs $\left(e_{1}, e_{2}\right)$ with $e_{j}$ in $E_{j}$ and $e_{1} \theta_{1}=e_{2} \theta_{2}$. (ii) $\left(e_{1}, e_{2}\right)=\left(e_{1}^{\prime}, e_{2}^{\prime}\right)$ if and only if $e_{1}^{\prime}=e_{1} g, e_{2}^{\prime}=e_{2} g^{-1}$ for some $g$ in $G$. (iii) $\left(e_{1}, e_{2}\right)\left(e_{1}^{\prime}, e_{2}^{\prime}\right)=\left(e_{1} e_{1}^{\prime}, e_{2} e_{2}^{\prime}\right)$. (iv) $\left(e_{1}, e_{2}\right) \theta=e_{1} \theta_{1}=e_{2} \theta_{2}$. (v) $(g, 1)=g$ for $g$ in $G$. (Notation: $E_{1} \otimes E_{2}$ $=E$.)

For a more detailed discussion of the product see Eilenberg and MacLane [2, 3]. Straightforward but tedious calculation shows that $E_{1} \otimes E_{2}$ is a $(G, M)$ extension such that

$$
\text { If } \begin{aligned}
E_{j} \sim E_{j}^{\prime}(j=1,2), & E_{1} \otimes E_{2} \sim E_{1}^{\prime} \otimes E_{2}^{\prime}, \\
E_{1} \otimes E_{2} & \sim E_{2} \otimes E_{1}, \\
\left(E_{1} \otimes E_{2}\right) \otimes E_{3} & \sim E_{1} \otimes\left(E_{2} \otimes E_{3}\right) .
\end{aligned}
$$

Therefore the set $S$ of all $(G, M)$ extensions, with equivalence as equality, and with multiplication as in Definition 5, is a commutative semigroup. It may also be shown that $S$ has a unit $\left(E_{o}, \theta_{o}\right)$ :

Definition 6. The unit extension $\left(E_{o}, \theta_{o}\right)$ is defined as follows: $E_{o}$ is the set of all pairs $(x, g), x$ in $M, g$ in $G$, such that (i) $(x, g)$ 
$=\left(y, g^{\prime}\right)$ if and only if $x=y, g=g^{\prime}$; (ii) $(x, g)\left(y, g^{\prime}\right)=\left(x y,(g y) g^{\prime}\right)$; (iii) $(1, g)=g$. And $\theta_{0}$ is given by (iv) $(x, g) \theta_{0}=x$.

It is essentially known (Baer [1], Eilenberg-MacLane [1]) that the subset $S^{\prime}$ of $S$, consisting of the central extensions, is an abelian group with unit $\left(E_{o}, \theta_{o}\right)$. For $(E, \theta)$ central, our inverse $(E, \theta)^{-1}$ is the inverse of $(E, \theta)$ in $S^{\prime}$. Details are deferred until $\$ 6$ (see Theorem 10) but the facts are assumed in $\$ 4$.

2. The functions $F$. For any positive integer $n$ let $L_{n}$ be the free loop (Bates [1]) with (free) generators $X_{1}, \cdots, X_{n}$. Thus $L_{n}$ is a loop containing the $X_{j}$, such that any mapping $X_{1} \rightarrow e_{1}, \ldots, X_{n} \rightarrow e_{n}$ into elements $e_{j}$ of a loop $E$ may be extended uniquely to a homomorphism $\rho$ of $L_{n}$ into $E$. By a (nonassociative) word $W_{n}$ we mean any element of $L_{n}$. The image $W_{n} \rho$ is denoted by $W_{n}\left(e_{1}, \cdots, e_{n}\right)$; this turns $W_{n}$ into a function defined on every loop $E$ (with values in $E)$. The following fact is worth noting: if also $\sigma$ is a homomorphism of $E$ into a loop $L, W_{n}\left(e_{1}, \cdots, e_{n}\right) \sigma=W_{n}\left(e_{1} \sigma, \cdots, e_{n} \sigma\right)$, since the homomorphism $\rho \sigma$ of $L_{n}$ maps $X_{j}$ upon $e_{j} \sigma$.

Definition 7. A word $W_{n}$ is purely nonassociative (p.n.a.) if it "vanishes" on every group: If $e_{1}, \cdots, e_{n}$ are group elements,

$$
W_{n}\left(e_{1}, \cdots, e_{n}\right)=1 \text {. }
$$

As an important example of a p.n.a. word, consider $A_{3}$, defined by $\left(X_{1} X_{2}\right) X_{8}=\left(X_{1}\left(X_{2} X_{8}\right)\right) A_{3}\left(X_{1}, X_{2}, X_{3}\right)$. If $E$ is a loop, the set of all elements $W_{n}\left(e_{1}, \ldots, e_{n}\right)$ ( $n$ arbitrary, $W_{n}$ p.n.a., the $e_{j}$ in $E$ ) generates a normal subloop $E_{p n a}$ which may be characterized as follows: a necessary and sufficient condition that $E / F$ be associative (for a normal subloop $F$ of $E$ ) is that $F$ contain $E_{\text {pna }}$.

THEOREM 1. Let $(E, \theta)$ be $a(G, M)$ extension, $W_{n}, a$ p.n.a. word, $e_{1}, \cdots, e_{n}$, elements of $E$. Write $e_{j} \theta=x_{j}, e_{0}=W_{n}\left(e_{1}, \cdots, e_{n}\right)$. Then (i) $e_{o} k=k e_{o}$ for $k$ in the kernel $K$; (ii) $W_{n}\left(x_{1}, \cdots, x_{n}\right)=1$ if and only if $e_{0}$ is in $G$; (iii) $e_{0}$ depends only on the $x_{j}$ :

$$
e_{o}=W_{n}\left(e_{1}, \cdots, e_{n}\right)=F\left(W_{n}, E ; x_{1}, \cdots, x_{n}\right) .
$$

Proof. (i) If $T$ is defined by (1), the mapping $e \rightarrow T(e)$ is a homomorphism of $E$ upon a group of automorphisms of $K$. Thus $T\left(e_{o}\right)$ $=W_{n}\left(T\left(e_{1}\right), \cdots, T\left(e_{n}\right)\right)=1$, the identity automorphism.

(ii) $e_{0} \theta=W_{n}\left(x_{1}, \cdots, x_{n}\right)$, so (i) implies (ii).

(iii) For fixed $n$, and for every word $A_{n}$ (not necessarily p.n.a.), define a function $H\left(A_{n} ; e, k\right)=H\left(A_{n} ; e_{1}, \cdots, e_{n} ; k_{1}, \ldots, k_{n}\right)$ by

$$
A_{n}\left(e_{1} k_{1}, \cdots, e_{n} k_{n}\right)=A_{n}\left(e_{1}, \cdots, e_{n}\right) H\left(A_{n} ; e, k\right) .
$$


Here the $e_{j}$ are assigned fixed values in $E$ and the $k_{j}$ vary over $K$. Applying $\theta$ to (6) we find that $H$ takes values in $K$. Also from (6), direct computation, along with the fact that $\left(A_{n} B_{n}\right)\left(e_{1}, \cdots, e_{n}\right)$ $=A_{n}\left(e_{1}, \cdots, e_{n}\right) B_{n}\left(e_{1}, \cdots, e_{n}\right)$, gives

$$
H\left(A_{n} B_{n} ; e, k\right)=H\left(A_{n} ; e, k\right) T\left(B_{n}\left(e_{1}, \cdots, e_{n}\right) \cdot H\left(B_{n} ; e, k\right) .\right.
$$

Moreover, by specializing $A_{n}$ in (6) to the "unit" word 1 and the words $X_{j}$,

$$
H(1 ; e, k)=1 ; \quad H\left(X_{j} ; e, k\right)=k_{j} \quad(j=1,2, \cdots, n) .
$$

In addition, if $B_{n} C_{n}=A_{n}=D_{n} B_{n}$, we may derive from (7) formulas involving only $A_{n}$ and $C_{n}$ or $A_{n}$ and $D_{n}$. Hence, since $L_{n}$ is free, the recurrence formula (7) and the initial conditions (8) define a unique function $H$.

Next construct the holomorph $\Re$ of $K$. This group is the set of all pairs $(S, k), k$ in $K, S$ an automorphism of $K$, under the product $(S, k)\left(U, k^{\prime}\right)=\left(S U, k U \cdot k^{\prime}\right)$. The $n$ elements $f_{j}=\left(T\left(e_{j}\right), k_{j}\right)$ yield $A_{n}\left(f_{1}, \cdots, f_{n}\right)=\left(T\left(A_{n}\left(e_{1}, \cdots, e_{n}\right)\right), H^{\prime}\left(A_{n} ; e, k\right)\right)$ where $H^{\prime}$ satisfies both (7) and (8). Therefore $H=H^{\prime}$. Since $\Omega$ is a group, $H^{\prime}\left(W_{n}\right.$; $e, k)=1$ for every p.n.a. word $W_{n}$. Thus, by (6), $W_{n}\left(e_{1} k_{1}, \cdots, e_{n} k_{n}\right)$ $=W_{n}\left(e_{1}, \cdots, e_{n}\right)=e_{o}$, showing that $e_{o}$ depends only on the images $x_{j}=e_{j} \theta=\left(e_{j} k_{j}\right) \theta$. This completes the proof of Theorem 1 .

Definition 8 . An ordered set $x_{1}, \cdots, x_{n}$ of elements of $M$ is called a spot for a p.n.a. word $W_{n}$ if $W_{n}\left(x_{1}, \cdots, x_{n}\right)=1$.

THEOREM 2. At each spot for a p.n.a. word $W_{n}$, the functions $F$ (of Theorem 1) form a multiplicative abelian group: (i) $E_{1} \sim E_{2}$ implies $F\left(W_{n}, E_{1}\right)=F\left(W_{n}, E_{2}\right)$; (ii) $E_{1} \sim^{-1} E_{2}$ implies $F\left(W_{n}, E_{1}\right)=F\left(W_{n}, E_{2}\right)^{-1}$; (iii) $F\left(W_{n}, E_{1}\right) F\left(W_{n}, E_{2}\right)=F\left(W_{n}, E_{1} \otimes E_{2}\right)$.

Proof. Let $x_{1}, \cdots, x_{n}$ be a spot for $W_{n}$, and write $F\left(W_{n}, E\right)$ $=F\left(W_{n}, E ; x_{1}, \cdots, x_{n}\right)$ for any extension $(E, \theta)$. By Theorem 1 (ii), $F\left(W_{n}, E\right)$ is in $G$. Let $\pi$ be an isomorphism of $\left(E_{1}, \theta_{1}\right)$ upon $\left(E_{2}, \theta_{2}\right)$ satisfying (i) of Definitions 3,4 , and let $e_{j}$ in $E_{1}$ satisfy $e_{j} \theta_{1}$ $=x_{j}(j=1,2, \ldots, n)$. Then $e_{j} \pi$ is in $E_{2}$, and $e_{j} \pi \theta_{2}=e_{j} \theta_{1}=x_{j}$. Hence $F\left(W_{n}, E_{1}\right) \pi=W_{n}\left(e_{1}, \cdots, e_{n}\right) \pi=W_{n}\left(e_{1} \pi, \cdots, e_{n} \pi\right)=F\left(W_{n}, E_{2}\right)$. According as $\pi$ satisfies (ii) of Definition 3 or 4 , we get (i) or (ii) of Theorem 2. To prove (iii), choose $e_{1 j}$ in $E_{1}, e_{2 j}$ in $E_{2}$ such that $e_{1 j} \theta_{1}=e_{2 j} \theta_{2}=x_{j}$, and set $e_{j}=\left(e_{1 j}, e_{2 j}\right),(j=1,2, \cdots, n)$. If $g_{i}=F\left(W_{n}, E_{i}\right)$, Definition 5 gives $F\left(W_{n}, E_{1} \otimes E_{2}\right)=W_{n}\left(e_{1}, \cdots, e_{n}\right)=\left(g_{1}, g_{2}\right)=\left(g_{1} g_{2}, 1\right)=g_{1} g_{2}$ $=F\left(W_{n}, E_{1}\right) F\left(W_{n}, E_{2}\right)$.

3. Strongly grouplike and $C$ extensions. An extension $(E, \theta)$ is strongly grouplike (s.g.) if $E$ inherits all relations between elements 
(implied by the associative law) which hold for the images in $M$. This means: if $W_{n}$ is p.n.a., and if $W_{n}\left(e_{1}, \cdots, e_{n}\right) \theta=1$, then $W_{n}\left(e_{1}, \cdots, e_{n}\right)$ $=1$. In particular, if $M$ is a group, the s.g. extensions are precisely the associative extensions. The following theorem is an immediate consequence of Theorem 2.

Theorem 3. (i) For any $(G, M)$ extension $E, E \otimes E^{-1}$ is s.g. (ii) If $E$ is s.g., and if $E_{1} \sim E$ or $E_{1} \sim^{-1} E$, then $E_{1}$ is s.g. (iii) If $E_{1} \otimes E_{2}=E_{3}$, and if two of the $E_{j}$ are s.g., so is the third.

Next let $C$ be any set of p.n.a. words. Assume that if $W_{n}$ is in $C$ then $W_{n}\left(x_{1}, \cdots, x_{n}\right)=1$ for all $x_{j}$ in $M$. Then a $(G, M)$ extension $(E, \theta)$ is " $C$ " if $W_{n}\left(e_{1}, \cdots, e_{n}\right)=1$ for each $W_{n}$ in $C$ and all $e_{j}$ in $E$. We get at once the following theorem.

Theorem 4. Every s.g. extension is $C$, and Theorem 3 remains true with "s.g." replaced by " $C$ ".

The following examples are of interest: (1) $C$ consists of $A_{3}$, introduced after Definition 7. $M$ is a group and the $C$-extensions are the associative ones. (2) $C$ consists of $B_{3}$, defined by $X_{1} X_{2} \cdot X_{3} X_{1}$ $=\left(X_{1}\left(X_{2} X_{3} \cdot X_{1}\right)\right) B_{3}\left(X_{1}, X_{2}, X_{3}\right) . M$ is a Moufang loop (Bruck [1]), characterized by the identity

$$
x y \cdot z x=x(y z \cdot x),
$$

and the $C$-extensions are the Moufang ones.

4. Groups of extensions. First let $S$ be any commutative semigroup. A subset $N$ is a nucleus of $S$ if there exists a homomorphism $\rho$ of $S$, with kernel $N$, upon a group. Equivalently: (i) if $a_{1} a_{2}=a_{3}$ for $a_{j}$ in $S$, and if two of the $a_{j}$ are in $N$, so is the third; (ii) to each $a$ in $S$ corresponds an $a^{-1}$ in $S$ such that $a a^{-1} \in N$. The necessity of (i), (ii) is obvious. As for sufficiency, define $a \equiv b \bmod N$ if $a n_{1}=b n_{2}$ for $n_{j}$ in $N$, and let $a \rho$ be the equivalence class of $a \bmod N$; then $\rho$ is a homomorphism, with kernel $N$, of $S$ upon the quotient group $S \rho=S / N$. If the nucleus $N^{\prime}$ contains the nucleus $N$, one may establish the isomorphism $S / N^{\prime} \cong(S / N) /\left(N^{\prime} / N\right)$. Furthermore, if $S$ has a unit contained in a subgroup $S^{\prime}$ of $S$, then $N S^{\prime}$ is a nucleus and one may establish the isomorphism $\left(N S^{\prime}\right) / N \cong S^{\prime} /(S \cap N)$. These remarks lead to the following (restricted) definition.

Definition 9. A subset $N$ of the semigroup $S$ of $(G, M)$ extensions (or of the group $S^{\prime}$ of central extensions) is a nucleus of $S$ (or $S^{\prime}$ ) provided (i) if $E_{1} \otimes E_{2}=E_{3}$ for (central) extensions $E_{j}$, and if two of the $E_{j}$ are in $N$, so is the third; (ii) for every (central) extension $E$, $E \otimes E^{-1}$ is in $N$, where $E^{-1}$ denotes the inverse extension. 
The following are nuclei of $S$ : (i) the set $N_{s g}$ of s.g. extensions (Theorem 3); (ii) the set $N_{C}$ of $C$-extensions (Theorem 4); (iii) $S^{\prime} \otimes N_{s g}$; (iv) $S^{\prime} \otimes N_{C}$. As nuclei of $S^{\prime}$ we have the subgroups $N_{s g}^{\prime}$ $=S^{\prime} \cap N_{s g}, N_{C}^{\prime}=S^{\prime} \cap N_{C}$. We define abelian groups $\mathbb{3}, \mathfrak{B}, \mathfrak{S}$ by

$$
\mathbb{B}=S / N_{s g}, \quad \mathfrak{B}=\left(S^{\prime} \otimes N_{s g}\right) / N_{s o} \cong S^{\prime} / N^{\prime}{ }_{s q}, \quad \mathfrak{E}=\mathbb{B} / \mathfrak{B} \text {. }
$$

Similar definitions hold for $\mathfrak{B}_{c}, \mathfrak{B}_{c}, \mathfrak{E}_{c}$. In view of Theorem 2, these groups are isomorphic to certain groups of functions $F$. A characterization of the latter would be highly enlightening. So far, however, not much is known. At the one end of the scale we have the following theorem.

THEOREM 5. If the loop $M$ is free, $\mathfrak{Z}, \mathfrak{B}$, and $\mathfrak{S}$ are groups of order 1.

Proof. Let $(E, \theta)$ be a $(G, M)$ extension. In particular, $\theta$ is a homomorphism of $E$ upon $M$. Since $M$ is free, there exists (Bates [1, Theorem 3.5]) an isomorphism $\rho$ of $M$ into $E$ such that $x \rho \theta=x$ for each $x$ in $M$. Let $W_{n}$ be any p.n.a. word, $x_{1}, \cdots, x_{n}$ any spot for $W_{n}$. Then $F\left(W_{n}, E ; x_{1}, \cdots, x_{n}\right)=W_{n}\left(x_{1} \rho, \cdots, x_{n} \rho\right)=W_{n}\left(x_{1}, \cdots, x_{n}\right) \rho$ $=1 \rho=1$. Therefore $S=N_{s g}$, which implies Theorem 5 .

A similar result holds for $C$-extensions. Define a loop $L$ to be a $C$-loop if $W_{n}\left(y_{1}, \cdots, y_{n}\right)=1$ for every $W_{n}$ in $C$ and all $y_{1}, \cdots, y_{n}$ in $L$. By previous agreement, $M$ is a $C$-loop, and $E$ is a $C$-loop for every $C$-extension $(E, \theta)$. The notion of a free $C$-loop may be defined as in Bates [1, Appendix]. Restricting attention to $C$-extensions, the proof of Theorem 5 may be paralleled exactly to give the following theorem.

Theorem 6. If $M$ is a free C-loop, $N_{C}=N_{s g}$. In words: the $C$-extensions coincide with the strongly grouplike extensions.

At the other end of the scale, take $M$ to be a group. For $n \geqq 0$, a (normalized) n-cochain $f_{n}$ is (Eilenberg and MacLane $[1,2,3]$ ) a single-valued function from $M$ to $G$, with values $f_{n}\left(x_{1}, \ldots, x_{n}\right)$, taking the value 1 if at least one of the $x_{j}$ is 1 . These $n$-cochains form the $n$-cochain group $\mathfrak{E}_{n}$ under the product $\left(f_{n} h_{n}\right)\left(x_{1}, \ldots, x_{n}\right)=$ $f_{n}\left(x_{1}, \cdots, x_{n}\right) h_{n}\left(x_{1}, \cdots, x_{n}\right)$. We define the $(n+1)$-coboundary $\delta f_{n}$ of $f_{n}$ as the normalized cochain

$$
\begin{array}{r}
\left(\delta f_{n}\right)\left(x_{1}, \cdots, x_{n+1}\right)=\left(f_{n}\left(x_{1}, \cdots, x_{n}\right) x_{n+1}\right) \cdot f_{n}\left(x_{2}, \cdots, x_{n+1}\right)^{c(0)} \\
\cdot \prod_{i=1}^{n} f_{n}\left(x_{1}, \cdots, x_{i-1}, x_{i} x_{i+1}, x_{i+2}, \cdots, x_{n+1}\right)^{c(i)}
\end{array}
$$

where $c(j)=(-1)^{n+1+j}$ for $j=0,1, \cdots, n$. For $n>0, \mathfrak{B}_{n}$ is the group 
of the $n$-coboundaries; $\mathfrak{B}_{0}$ consists of the 0 -cochain $1_{0}=1$. An $n$-cocycle is an $n$-cochain $f_{n}$ such that $\delta f_{n}=1_{n+1}$ (the identity of $\mathfrak{G}_{n+1}$ ) and $Z_{n}$ is the group of the $n$-cocycles. As a consequence of the associativity of $M$, one may verify that $\delta^{2}=0$, in the sense that $\delta\left(\delta f_{n}\right)=1_{n+2}$; hence $\mathfrak{B}_{n}$ is a subgroup of $\mathfrak{Z}_{n}$. The $n$th cohomology group $\mathfrak{S}_{n}$ is defined by $\mathfrak{S}_{n}=\mathfrak{B}_{n} / \mathfrak{B}_{n}$. The next theorem is due to Eilenberg and MacLane $[2,3]$ :

THEOREM 7. If $M$ is a group, the homomorphism $(E, \theta) \rightarrow F\left(A_{8}, E\right)$ induces the isomorphism $\mathfrak{S} \cong \mathfrak{S}_{3}$.

A partial sketch of the proof will be useful. For any $(G, M)$ extension $(E, \theta)$, define (see $\S 3) f_{a}$ and $f_{m}$ by

$$
f_{a}(x, y, z)=F\left(A_{3}, E ; x, y, z\right) ; f_{m}(x, y, z)=F\left(B_{3}, E ; x, y, z\right) .
$$

Choose $e_{j}$ in $E$ such that $e_{j} \theta=x_{j}$ for $j=1,2,3,4$. Then

(13) $\left(e_{1} e_{2}\right) e_{3}=e_{1}\left(e_{2} e_{3}\right) f_{a}\left(x_{1}, x_{2}, x_{3}\right) ; e_{1} e_{2} \cdot e_{3} e_{1}=e_{1}\left(e_{2} e_{3} \cdot e_{1}\right) f_{m}\left(x_{1}, x_{2}, x_{3}\right)$,

showing that $f_{a}, f_{m}$ are normalized 3-cochains. If $(E, \theta)$ is central and if $u(x)$ is a normalized system of representatives of $M$ in $E$, then $u(x) u(y)=u(x y) h(x, y)$ for a normalized 2-cochain $h$. Setting $e_{j}=u\left(x_{j}\right)$ in (13) we find $f_{a}=\delta h$. In any case, by (13), $\left(e_{1} e_{2} \cdot e_{3}\right) e_{4}=\left(e_{1} e_{2} \cdot e_{3} e_{4}\right)$ $\cdot f_{a}\left(x_{1} x_{2}, x_{3}, x_{4}\right)=\left(e_{1}\left(e_{2} \cdot e_{3} e_{4}\right)\right) f_{a}\left(x_{1}, x_{2}, x_{3} x_{4}\right) f_{a}\left(x_{1} x_{2}, x_{3}, x_{4}\right)$ and also

$$
\begin{aligned}
\left(e_{1} e_{2} \cdot e_{3}\right) e_{4} & =\left(\left(e_{1} \cdot e_{2} e_{3}\right) f_{a}\left(x_{1}, x_{2}, x_{3}\right)\right) e_{4}=\left(e_{1} \cdot e_{2} e_{3}\right) e_{4}\left(f_{a}\left(x_{1}, x_{2}, x_{8}\right) x_{4}\right) \\
& =e_{1}\left(e_{2} e_{3} \cdot e_{4}\right) f_{a}\left(x_{1}, x_{2} x_{3}, x_{4}\right)\left(f_{a}\left(x_{1}, x_{2}, x_{3}\right) x_{4}\right) \\
& =\left(e_{1}\left(e_{2} \cdot e_{3} e_{4}\right)\right) f_{a}\left(x_{2}, x_{3}, x_{4}\right) f_{a}\left(x_{1}, x_{2} x_{3}, x_{4}\right)\left(f_{a}\left(x_{1}, x_{2}, x_{3}\right) x_{4}\right),
\end{aligned}
$$

whence comparison gives $\delta f_{a}=1_{4}$. Thus $f_{a}$ is a 3-cocycle. It can be shown conversely that every 3-cocycle (3-coboundary) is an $F\left(A_{3}, E\right)$ (an $F\left(A_{3}, E\right)$ for $E$ central).

Again, $e_{1} e_{2} \cdot e_{3} e_{1}=e_{1}\left(e_{2} \cdot e_{3} e_{1}\right) f_{a}\left(x_{1}, x_{2}, x_{3} x_{1}\right)$ and $e_{1}\left(e_{2} e_{3} \cdot e_{1}\right)=e_{1}\left(e_{2} \cdot e_{3} e_{1}\right)$ $f_{a}\left(x_{1}, x_{2}, x_{3}\right)$, whence, by (13),

$$
f_{m}(x, y, z)=f_{a}(x, y, z x) f_{a}(y, z, x)^{-1} .
$$

The homomorphism $\rho$ of $\boldsymbol{B}_{3}$ into $\mathfrak{E}_{3}$, defined by $\left(f_{3} \rho\right)(x, y, z)=f_{8}(x, y, z x)$ $\cdot f_{3}(y, z, x)^{-1}$, induces a homomorphism of $\mathfrak{S}_{3}$ upon a group $\mathfrak{S}_{3} \rho$ $=\mathfrak{B}_{3} \rho / \mathfrak{B}_{3} \rho$. In view of (14) we may state the following theorem.

Theorem 8. If $M$ is a group, let $C$-extensions be Moufang extensions. Then the homomorphism $(E, \theta) \rightarrow F\left(B_{3}, E\right)$ induces an isomorphism $\mathfrak{S}_{C} \cong \mathfrak{S}_{3} \rho$.

Theorems 5-8 have analogues for central extensions, for example (Baer [1], Eilenberg and MacLane [1]): if $M$ is a group, the group of 
central group extensions is isomorphic to the second cohomology group \$2.

5. Grouplike extensions. Conjugate extensions. $\mathrm{A}(G, M)$ extension $(E, \theta)$ is grouplike if, for every subgroup (=associative subloop) $H$ of $M, H \theta^{-1}$ is a subgroup of $E$. Thus $(E, \theta)$ is grouplike if and only if $F\left(A_{3}, E ; x, y, z\right)=1$ for all triples $x, y, z$ which generate a subgroup of $M$. Note that s.g. extensions are grouplike.

If $E$ is any loop, define for each $p$ in $E$ permutations $R_{p}, L_{p}$ by $e R_{p}=e p, e L_{p}=p e$, all $e$ in $E$. Choosing fixed $p, q$ in $E$, we may define a new operation (o) on $E$ by

$$
e_{1} 0 e_{2}=\left(e_{1} R_{q}^{-1}\right)\left(e_{2} L_{p}^{-1}\right) \text {. }
$$

The elements of $E$ form a loop $E_{o}$ under $(0)$; the unit is $p q . E_{o}$ is (Albert [1]) a (principal) isotope of $E$. If, further, $(E, \theta)$ is a $(G, M)$ extension, write $p \theta=u, q \theta=v$. Then, if $M_{0}$ is the principal isotope of $M$ defined by

$$
x \circ y=\left(x R_{v}^{-1}\right)\left(y L_{u}^{-1}\right),
$$

we find from (15), (16), with $e_{j} \theta=x_{j}$, that $\left(e_{1} o e_{2}\right) \theta=x_{1} \circ x_{2}$.

For each $a$ in the associator $A(M)$, and for each $(G, M)$ extension $(E, \theta)$, define a loop $E^{a}$ as follows: Choose $p$ in $E$ so that $p \theta=a^{-1}$, and $q$ in $E$ so that $p q=1$. Then $E^{a}$ is the loop $E_{o}$ given by (15). We define $(E, \theta)^{a}=\left(E^{a}, \theta\right)$ to be a conjugate of $(E, \theta)$.

Theorem 9. Let $(E, \theta)$ be $a(G, M)$ extension, and let $a, b$ be in $A(M)$. Then: (i) $E^{a}$ is independent of the choice of $p$ in its definition; (ii) $\left(E^{a}, \theta\right)$ is a $(G, M)$ extension; (iii) $E_{1} \sim E_{2}$ implies $E_{1}^{a} \sim E_{2}^{a}$; (iv) $E_{1} \sim{ }^{-1} E_{2}$ implies $E_{1}^{a} \sim^{-1} E_{2}^{a}$; (v) $\left(E^{a}\right)^{b} \sim E^{a b}$; (vi) $\left(E_{1} \otimes E_{2}\right)^{a} \sim E_{1}^{a} \otimes E_{2}^{a}$.

Proof. (i) In (15), $p q=1$. Clearly we can construct a word $W_{3}$, independent of the loop $E$, so that (15) becomes $e_{1} 0 e_{2}=e_{1} e_{2} \cdot W_{3}\left(e_{1}, e_{2}, p\right)$. If $E$ is a group, (15) yields $e_{1} 0 e_{2}=\left(e_{1} q^{-1}\right)\left(p^{-1} e_{2}\right)=e_{1} p p^{-1} e_{2}=e_{1} e_{2}$; thus $W_{8}$ is p.n.a. Since, in (16), $u=p \theta=a^{-1}, v=q \theta=a$, with $a$ in $A(M)$, we have $x o y=x a^{-1} \cdot\left(a^{-1}\right)^{-1} y=x\left(a^{-1} \cdot a y\right)=x y$. Hence $W_{3}\left(e_{1}, e_{2}, p\right) \theta$ $=W_{3}\left(x_{1}, x_{2}, a^{-1}\right)=1$. By Theorem 1, $W_{3}\left(e_{1}, e_{2}, p\right)$ lies in $G$ and depends only on $x_{1}, x_{2}, a$.

(ii) Since $x o y=x y, \theta$ is a homomorphism of $E^{a}$ upon $M$. The kernel of $\theta$ (in $E^{a}$ ) is the subloop $K_{0}$ consisting of $K$ under (o). Since $p q=1$ is the unit of $E^{a}, W_{3}(1, e, p)=1=W_{3}(e, 1, p)$ for all $e$ in $E^{a}$. Hence, for $k$ in $K, e o k=e k W_{8}(e, k, p)=e k W_{8}(e, 1, p)=e k$ and $\left(e_{1} 0 e_{2}\right) o k$ $=\left(e_{1} 0 e_{2}\right) k=e_{1} e_{2} W_{3}\left(e_{1}, e_{2}, p\right) k=e_{1} e_{2} k W_{3}\left(e_{1}, e_{2}, p\right)=e_{1} o\left(e_{2} k\right)=e_{1} o\left(e_{2} 0 k\right)$. Similarly $\left(e_{1} o k\right) o e_{2}=e_{1}\left(k o e_{2}\right),\left(k o e_{1}\right) o e_{2}=k o\left(e_{1} o e_{2}\right)$, so that $K_{0}$ is in 
$A\left(E^{a}\right)$. The element $c$ of $K_{o}$ is in $Z\left(K_{o}\right)$ if and only if $c o k=k o c, c k=k c$, $c$ is in $G=Z(K)$. If $g_{1}, g_{2}$ are in $G, g_{1} 0 g_{2}=g_{1} g_{2} ;$ thus $G=Z\left(K_{o}\right)$. Finally, for $g$ in $G, e$ in $E, x=e \theta, g o e=g e=e(g x)=e o(g x)$. This completes the proof that $\left(E^{a}, \theta\right)$ is a $(G, M)$ extension.

(v) Assume $E^{a}=E_{o}$ is defined by (15), with $p \theta=a^{-1}, p q=1$. Then $\left(E^{a}\right)^{b}=E_{o}^{b}$ must be defined, with operation $(*)$, by $e_{1} * e_{2}=\left(e_{1} T\right) o\left(e_{2} S\right)$ $=\left(e_{1} T R_{q}^{-1}\right)\left(e_{2} S L_{p}^{-1}\right)$, where $e S^{-1}=s o e=\left(s R_{a}^{-1}\right)\left(e L_{p}^{-1}\right), e T^{-1}=e o t$ $=\left(e R_{q}^{-1}\right)\left(t L_{p}^{-1}\right)$ for $s, t$ in $E$ such that $s \theta=b^{-1}, 1=\operatorname{sot}=\left(s R_{q}^{-1}\right)\left(t L_{p}^{-1}\right)$. The elements $f=s R_{g}^{-1}, h=t L_{p}^{-1}$ satisfy $f \theta=b^{-1} a^{-1}, f h=1$. Moreover, $S L_{p}^{-1}=L_{f}^{-1}$ and $T R_{a}^{-1}=R_{h}^{-1}$. Therefore $e_{1} * e_{2}=\left(e_{1} R_{h}^{-1}\right)\left(e_{2} L_{j}^{-1}\right)$, showing that $\left(E^{a}\right)^{b}=E^{a b}$. The proofs of (iii), (iv), (vi) offer no difficulty, hence are omitted.

6. Central and central Moufang extensions. For any pair $(G, M)$ we may define the groups $\mathfrak{E}_{n}, \mathfrak{B}_{n}$ of (normalized) $n$-cochains and $n$ coboundaries. By (11), the $n$-coboundaries for $n=2,3$ are given by

$$
\begin{aligned}
\left(\delta f_{1}\right)(x, y) & =\left(f_{1}(x) y\right) f_{1}(y) f_{1}(x y)^{-1}, \\
\left(\delta f_{2}\right)(x, y, z) & =\left(f_{2}(x, y) z\right) f_{2}(y, z)^{-1} f_{2}(x y, z) f_{2}(x, y z)^{-1} .
\end{aligned}
$$

If $M$ is not associative we lose the important property $\delta^{2}=0$; in particular,

$$
\left(\delta^{2} f_{1}\right)(x, y, z)=f_{1}(x \cdot y z) f_{1}(x y \cdot z)^{-1} .
$$

Definition 10. Let $f, h$ be normalized 2-cochains of $(G, M)$. Then $f$ is equivalent to $h$ if $f=h \cdot \delta c$ for some (normalized) 1-cochain $c$. (Notation: $f \sim h$.)

Definition 11. If $f$ is a normalized 2-cochain of $(G, M)$, then $(G, M, f)$ is the central $(G, M)$ extension $(E, \theta)$ defined as follows: (i) The elements of $E$ are the pairs $(x, g), x$ in $M, g$ in $G$. (ii) $(x, g)$ $=\left(y, g^{\prime}\right)$ if and only if $x=y, g=g^{\prime}$. (iii) $(x, g)\left(y, g^{\prime}\right)=(x y, f(x, y)$ $\left.\cdot(g y) g^{\prime}\right)$. (iv) $(x, g) \theta=x$. (v) $(1, g)=g$.

By Definition 6, the unit extension $\left(E_{o}, \theta_{o}\right)$ may be identified with $(G, M, 1)$ where 1 is the identity 2 -cochain $1_{2}$.

THEOREM 10. (i) Each central $(G, M)$ extension is equivalent to at least one extension $(G, M, f)$. (ii) $(G, M, f) \sim(G, M, h)$ if and only if $f \sim h$. (iii) $(G, M, f) \sim^{-1}(G, M, h)$ if and only if $f \sim h^{-1}$. (iv) $(G, M, f)$ $\otimes(G, M, h) \sim(G, M, f h)$. (v) $(G, M, f)$ is grouplike if and only if $(\delta f)(x, y, z)=1$ for all $x, y, z$ which generate a subgroup of $M$. (vi) For a in $A(M),(G, M, f)^{a} \sim\left(G, M, f^{a}\right)$ where

$$
f^{a}(x, y)=f(x, y) \cdot(\delta f)\left(a^{-1}, a, y\right) \cdot\left((\delta f)\left(x a^{-1}, a, y\right)\right)^{-1} .
$$

Corollary. The set $S^{\prime}$ of central $(G, M)$ extensions is an abelian 
group with unit $\left(E_{o}, \theta_{0}\right)$ and inverse $(E, \theta)^{-1}$.

Proof. (i) Let $(E, \theta)$ be a central extension, $u(x)$ a normalized system of representatives of $M$ in $G$. Since $(u(x) u(y)) \theta=x y=u(x y) \theta$, $u(x) u(y)=u(x y) f(x, y)$ for $f(x, y)$ in $G$. Since $u(1)=1, f$ is a normalized 2-cochain. Every $e$ in $E$ has a unique representation $e=u(x) g$ with $g$ in $G, x=e \theta$. Moreover $u(x) g \cdot u(y) g^{\prime}=u(x) u(y)(g y) g^{\prime}=u(x y) f(x, y)$ $\cdot(g y) g^{\prime}$. Hence the mapping $u(x) g \rightarrow(x, g)$ gives the equivalence of $(E, \theta)$ and $(G, M, f)$.

(v) In the notation of (i), consider the equality $u(x) u(y) \cdot u(z)$ $=u(x) \cdot u(y) u(z)$.

(vi) In view of Theorem $9, E^{a}$ may be defined by $e_{1} o e_{2}=\left(e_{1} R_{q}^{-1}\right)$ $\cdot\left(e_{2} L_{p}^{-1}\right)$ where $p=u\left(a^{-1}\right)$ and $q=u(a) f\left(a^{-1}, a\right)^{-1}$. Write $u(x) o u(y)$ $=u(x y) h(x, y)$, so that $h=f^{a}$. Let $P=\left(u\left(x a^{-1}\right) q\right) o(p u(a y))$. On the one hand, $P=\left(u\left(x a^{-1}\right) R_{q} R_{a}^{-1}\right)\left(u(a y) L_{p} L_{p}^{-1}\right)=u\left(x a^{-1}\right) u(a y)=u\left(x a^{-1}\right.$ -ay) $f\left(x a^{-1}, a y\right)=u(x y) f\left(x a^{-1}, a y\right)$. On the other hand, since $u\left(x a^{-1}\right) q$ $=u\left(x a^{-1}\right) \cdot u(a) f\left(a^{-1}, a\right)^{-1}=u(x) f\left(x a^{-1}, a\right) f\left(a^{-1}, a\right)^{-1}, p u(a y)=u\left(a^{-1}\right)$ $\cdot u(a y)=u(y) f\left(a^{-1}, a y\right)$ and $(u(x) g) o\left(u(y) g^{\prime}\right)=u(x y) h(x, y)(g y) g^{\prime}$, $P=u(x y) h(x, y)\left(f\left(x a^{-1}, a\right) y\right)\left(f\left(a^{-1}, a\right) y\right)^{-1} f\left(a^{-1}, a y\right)$. Comparison of the two expressions for $P$ gives $h(x, y)=f\left(x a^{-1}, a y\right)\left(f\left(a^{-1}, a\right) y\right)$ $\cdot\left(f\left(x a^{-1}, a\right) y\right)^{-1} f\left(a^{-1}, a y\right)^{-1}$. However, substitution from (18) in the right-hand side of (20) yields precisely this expression for $h=f^{a}$.

(ii), (iii), (iv). For $j=1,2$, denote the elements of $\left(E_{j}, \theta_{j}\right)=\left(G, M, f_{j}\right)$ by $(x, g)_{j}$, where $(x, g)_{j} \theta_{j}=x$. Set $u_{j}(x)=(x, 1)_{j}$. If $\pi$ is an isomorphism of $E_{1}$ upon $E_{2}$ such that $\pi \theta_{2}=\theta_{1}$, then necessarily $u_{1}(x) \pi=u_{2}(x) c(x)$ $=(x, c(x))_{2}$ for a normalized 1-cochain $c$; and $(x, g)_{1} \pi=(x,(g \pi) c(x))_{2}$. Also $g \pi x=g x \pi$. Conversely, if $\pi$ is any automorphism of $G$ (such that $g \pi x=g x \pi)$ and $c$ any normalized 1-cochain, the definition $(x, g)_{1} \pi$ $=(x,(g \pi) c(x))_{2}$ extends $\pi$ to an isomorphism of $E_{1}$ upon $E_{2}$ such that $\pi \theta_{2}=\theta_{1}$. Direct calculation gives $f_{1}(x, y) \pi=f_{2}(x, y) \cdot(\delta c)(x, y)$; (ii), (iii) come by assuming $g \pi=g, g \pi=g^{-1}$ respectively. For $E_{1} \otimes E_{2}$ take the representatives $u(x)=\left(u_{1}(x), u_{2}(x)\right)$; Definition 5 gives $u(x) u(y)$ $=\left(u_{1}(x y) f_{1}(x, y), u_{2}(x y) f_{2}(x, y)\right)=u(x y) f_{1}(x, y) f_{2}(x, y)$, proving (iv). The corollary should be obvious.

Note that if $c$ is a 1-cochain and if $a$ is in $A(M)$, (19) gives $\left(\delta^{2} c\right)\left(x a^{-1}, a, y\right)=c\left(x a^{-1} \cdot a y\right) c(x y)^{-1}=1$. Thus it is evident from (20) that the cochain $f^{a} f^{-1}$ is invariant under replacement of $f$ by an equivalent cochain. We now turn to Moufang loops.

Theorem 11. Let $M$ be a Moufang loop. Then: (i) $x y \cdot z x=x(y z \cdot x)$ for all $x, y, z$ in $M$. (ii) $x(y \cdot x z)=(x y \cdot x) z$ for all $x, y, z$ in $M$. (iii) Every loop $M_{o}$ isotopic to $M$ is Moufang. (iv) The subloop generated by any two elements $x, y$ of $M$ is a group. (v) If the three elements $x, y, z$ satisfy 
$x y \cdot z=x \cdot y z$, they generate an associative subloop. (vi) The central extension $(G, M, f)$ is Moufang if and only if $f$ satisfies one of the (equivalent) conditions for a Moufang cochain:

(21a) $f(x y, z x)(f(x, y) z x) f(z, x)=f(x, y z \cdot x) f(y z, x)(f(y, z) x)$;

(21b) $\quad f(x, y \cdot z x) \cdot(\delta f)(x, y, z x)=f(x, y z \cdot x) \cdot(\delta f)(y, z, x)$.

(vii) The central Moufang $(G, M)$ extensions form a subgroup of the group of central extensions. (viii) If $f$ is a Moufang cochain, (20) simplifies to

$$
f^{a}(x, y) f(x, y)^{-1}=(\delta f)\left(x a^{-1}, a, y\right)^{-1} ;
$$

in particular, for each a of $A(M)$, the 2-cochain defined by the right side of (22) is Moufang.

Proof. Items (i)-(v) are included for reference. For a proof that (i) and (ii) are equivalent, and for (iii), see Bruck [1, Chapter II]. Items (iv), (v) are due to Moufang [1]; see also Bruck [2]. As for (vi), the extension $E=(G, M, f)$ is Moufang if and only if the word $B_{3}$ of $\$ 3$ vanishes on $E$. Assuming $u(x) u(y)=u(x y) f(x, y)$, the condition $B_{3}(u(x), u(y), u(z))=1$ gives precisely (21a), which, by (18), is equivalent to (21b). (vii) follows from (21) and Theorem 10. As for (viii), the elements $u(x), u(y)$ of the Moufang loop $E$ generate a group, by (iv). Since $u(x)^{-1}=u\left(x^{-1}\right) g$ for some $g$ in $G$, the condition $u(x)^{-1} u(x) \cdot u(y)=u(x)^{-1} \cdot u(x) u(y)$ reduces to $\left(\delta f^{\prime}\right)\left(x^{-1}, x, y\right)=1$. In particular, (20) becomes (22). Since $E^{a} \otimes E^{-1} \sim\left(G, M, f^{a} f^{-1}\right)$, (iii), (vii) imply the concluding statement of (viii).

Theorem 12. Let $M$ be a finite Moufang loop of order $m$. Let the least common multiple of the orders of the elements of $M$ be $n$. For any $a$ in $A(M)$, and for any central Moufang $(G, M)$ extension $(E, \theta)$ : (i) $E^{a}$ is Moufang. (ii) $E^{m n} \sim E_{o}$. (iii) $\left(E^{a} \otimes E^{-1}\right)^{2 m}$ is grouplike. (iv) If $M$ is commutative, $E^{2 m}$ is grouplike. (v) If $n$ is odd, the exponent $2 m$ in (iii), (iv) may be replaced by $m$. (vi) If $g x=g$ for all $g$ in $G, x$ in $M$, $E^{m} \sim E_{o}$.

Proof. (i) reflects Theorem 11 (iii) and was used for (viii). For the proof of (ii)-(vi), take $(E, \theta)=(G, M, f)$ where $f$ satisfies (21). Define the following (normalized) cochains:

$$
c(x)=\prod_{y} f(x, y), \quad d(x)=\prod_{y} f(y, x),
$$

where the products are taken over the $m$ elements $y$ of $M$, and

$$
h(x, y)=(c(x) y) c(x)^{-1} \text {. }
$$


From (24),

$$
h(x, y z)=(h(x, y) z) h(x, z)
$$

This implies

$$
h(w, x y \cdot z)=h(w, x \cdot y z),
$$

since both sides reduce to $(h(w, x) y z)(h(w, y) z) h(w, z)$. If $f_{1}(x, y)$ $=h(y, x y)^{-1}$, we take products in (21a) over all $y$, use (23), (24) and find $f(z, x)^{m}=(\delta d)(z, x) \cdot f_{1}(z, x)$, or

$$
f^{m} \sim f_{1}, \quad f_{1}(x, y)=h(y, x y)^{-1} .
$$

If $g x=g$ for all $g, x, h=1$ by (24) and $f^{m} \sim 1$ by (27), proving Theorem 12 (vi).

Since $1=f_{1}(1, y)=h(y, y)^{-1}$, or $h(y, y)=1,(25)$ implies

$$
h(x, x)=1, \quad h(x, x y)=h(x, y), \quad h(x, y x)=h(x, y) x .
$$

Since (21a) applies to $f_{1}$, set $z=1$ and get $f_{1}(x y, x)\left(f_{1}(x, y) x\right)=f_{1}(x, y x)$ $\cdot f_{1}(y, x)$. Вy (27), (28), $f_{1}(x y, x)=h(x, x y x)^{-1}=h(x, y x)^{-1}=f_{1}(y, x)$, leaving $f_{1}(x, y) x=f_{1}(x, y x)=h(y x, x y x)^{-1}=(h(y x, x) y x)^{-1}$, or $f_{1}(x, y)$ $=(h(y x, x) y)^{-1}$. Thus $h(y x, x) y=f_{1}(x, y)^{-1}=h(y, x y)=h(y, x) y$, $h(y x, x)=h(y, x)$, or

$$
h(x y, y)=h(x, y) .
$$

Returning to (21a), take products over all $z$, getting

$$
\prod_{z}(f(x, y) z)=(c(y) x) c(x) c(x y)^{-1}=h(y, x) c(x) c(y) c(x y)^{-1} .
$$

The left-hand element of (30) remains fixed when we operate with $w$. Thus, by $(24),(h(y, x) w) h(y, x)^{-1} h(x, w) h(y, w) h(x y, w)^{-1}=1$; whence, by (25),

$$
h(y, x w) h(x, w)=h(y, x) h(x y, w) .
$$

Set $w=y$ in (31) and use (29). Thus $h(y, x y) h(x, y)=h(y, x) h(x y, y)$ $=h(y, x) h(x, y), h(y, x y)=h(y, x)$, and

$$
h(x, y x)=h(x, y) .
$$

In view of (28.3), (32), $h(x, y) x=h(x, y)$. Hence, by (29), $h(x, y) y$ $=h(x, y) x y=h(x y, y) x y=h(x y, y)=h(x, y)$. Therefore

$$
h(x, y) x=h(x, y) y=h(x, y) \text {. }
$$

From (29) with $y$ replaced by $x^{-1} y, h\left(y, x^{-1} y\right)=h\left(x, x^{-1} y\right)$. By (32) and (28.2), this implies $h\left(y, x^{-1}\right)=h(x, y)$. Then, by (33), (25), 
$h(x, y) h(y, x)=h\left(y, x^{-1}\right) h(y, x)=\left(h\left(y, x^{-1}\right) x\right) h(y, x)=h\left(y, x^{-1} x\right)$ $=h(y, 1)=1$, or

$$
h(y, x)^{-1}=h(x, y) .
$$

Hence (32), (34) give $f_{1}(x, y)=h(y, x y)^{-1}=h(y, x)^{-1}=h(x, y)$, so

$$
f_{1}=h \text {. }
$$

Since $h(x, y) y=h(x, y)$, a simple induction using (25) gives $h\left(x, y^{j}\right)$ $=h(x, y)^{j}$. Combining this with (34),

$$
h\left(x^{i}, y^{j}\right)=h(x, y)^{i j}
$$

for all integers $i, j$. In particular, $f_{1}(x, y)^{n}=h(x, y)^{n}=h\left(x, y^{n}\right)=h(x, 1)$ $=1$, and so $f^{m n} \sim f_{1}^{n}=1$. This proves Theorem 12 (ii).

If $p=\delta f_{1}=\delta h,(18)$ and (25) combine to give

$$
h(x y, z)=h(x, z) h(y, z) p(x, y, z), \quad \quad p=\delta h .
$$

Since $h$ satisfies (21b), (26),

$$
p(x, y, z x)=p(y, z, x) .
$$

Operating on (37) by $w$, and using (25), we find

$$
p(x, y, z w)=(p(x, y, z) w) p(x, y, w) .
$$

Again, since $h(x, z) z=h(x, z)$, (37) gives $p(x, y, z) z=p(x, y, z)$. Hence, by (38), $p(x, y, z x) x=p(y, z, x)=p(x, y, z x)$, or $p(x, y, z) x=p(x, y, z)$. Thus, finally, $p(x, y, z x) y=p(y, z, x) y=p(y, z, x)=p(x, y, z x)$, and

$$
p(x, y, z) w=p(x, y, z),
$$$$
w=x, y, z \text {. }
$$

Since $h(x y, x)=h(x, x y)^{-1}=h(x, y)^{-1}=h(y, x)$ and $h(x, x)=1,(37)$ with $z=x$ gives $p(x, y, x)=1$. Therefore, by (38), (39), (40), $p(x, y, z x)$ $=(p(x, y, z) x) p(x, y, x)=p(x, y, z)$, so that (38) becomes

$$
p(x, y, z)=p(y, z, x) .
$$

By (37), (24), and (25), $p(x, y, z)=h(z, x) h(z, y) h(z, x y)^{-1}=h(z, x)$ $\cdot(h(z, x) y)^{-1}$. Therefore, by (41), (34),

$$
p(x, y, z)=h(x, y)(h(x, y) z)^{-1}=p(y, x, z)^{-1} .
$$

By this and (37),

$$
h(x y, z) h(y x, z)^{-1}=p(x, y, z)^{2} .
$$

Hence, if $M$ is commutative, (43) gives $((\delta h)(x, y, z))^{2}=1$ for all $x, y, z$. In view of (19), the best we can say for $k=f^{2 m}$ is that $(\delta k)(x, y, z)=1$ for all $x, y, z$ such that $x y \cdot z=x \cdot y z$. By Theorems $11(\mathrm{v}), 10(\mathrm{v})$, this is 
precisely the condition that $E^{2 m}$ be grouplike. We have proved Theorem 12(iv).

Since $f^{m} \sim h$ and $p=\delta h$, we see from Theorem 11(viii) that $\left(E^{a} \otimes E^{-1}\right)^{2 m} \sim(G, M, q)$ where

$$
q(x, y)=p\left(x a^{-1}, a, y\right)^{-2} \text {. }
$$

Define the (normalized) 4-cochain $r$ by

$$
r(w, x, y, z)=(p(w, x, y) z) p(w, x, y)^{-1} .
$$

By (45), $r$ has the skew-symmetry (41), (42) of $p$ on its first three arguments. By (39),

$$
p(w, x, y z)=p(w, x, y) p(w, x, z) r(w, x, y, z) .
$$

By (34), (26), $h(w x \cdot y, z)=h(w \cdot x y, z)$. Expand each side of this last equation by $(38)$, in the form $h(w, z) h(x, z) h(y, z)$. Equate, and use (46) to get $r(y, z, w, x)=r(z, w, x, y)$, whence $r(z, w, y, x)=r(z, w, x, y)$ or

$$
r(w, x, y, z)=r(w, x, z, y) .
$$

By (47) and skew-symmetry, $r(w, x, y, z)=r(w, x, z, y)=r(x, z, w, y)$ $=r(x, z, y, w)=r(y, x, z, w)=r(y, x, w, z)=r(w, x, y, z)^{-1}$, or

$$
r(w, x, y, z)^{2}=1 \text {. }
$$

From (44), (46), (48), $q(x, y)^{-1}=p\left(a, y, x a^{-1}\right)^{2}=p(a, y, x)^{2} p\left(a, y, a^{-1}\right)^{2}$. Since $q(1, y)=1$, the second factor is 1 , and, by (42),

$$
q(x, y)^{-1}=p(x, y, a)^{2}=h(x, y)^{2}(h(x, y) a)^{-2} .
$$

Therefore, since $p=\delta h,(\delta q)(x, y, z)^{-1}=p(x, y, z)^{2}(p(x, y, z) a)^{-2}$. Hence, by $(45),(48),(\delta q)(x, y, z)=1$ for all $x, y, z$. This proves Theorem 12 (iii).

As for (v), since $h^{n}=1$, (37) gives $p^{n}=1$ and then (45) gives $r^{n}=1$. However, $r^{2}=1$, by (48). Hence, if $n$ is odd, $r=1$ and (iii) holds with $2 m$ replaced by $m$. A similar remark is true of (iv). This completes the proof of Theorem 12 .

Theorem 12 should be compared with the simpler result for groups (Marshall Hall [1]): If $M$ is a group of order $m$ and if $(E, \theta)$ is a central associative $(G, M)$ extension, $E^{m} \sim E_{o}$.

\section{BibLIOGRAPHY}

A. A. Albert

1. Quasigroups. I, Trans. Amer. Math. Soc. vol. 54 (1943) pp. 507-519.

2. Quasigroups. II, Trans. Amer. Math. Soc. vol. 55 (1944) pp. 401-419. 
R. H. BRUCK

\section{REINHOLd BAER}

1. Erweiterungen von Gruppen und ihren Isomorphismen, Math. Zeit. vol. 38 (1934) pp. 375-416.

2. Automorphismen von Erweiterungsgruppen, Actualites Scientifiques et Industrielles, no. 205, Paris, 1935.

3. Representations of groups as quotient groups. II. Minimal chains of a group, Trans. Amer. Math. Soc. vol. 53 (1943) pp. 348-389.

Grace E. Bates

1. Free loops and nets and their generalizations, Amer. J. Math. vol. 69 (1947) pp. 499-550.

R. H. BRUCK

1. Contributions to the theory of loops, Trans. Amer. Math. Soc. vol. 60 (1946) pp. 245-354.

2. On a theorem of $R$. Moufang. To appear in the Proceedings of the American Mathematical Society.

Max Deuring

1. Algebren, Ergebnisse der Mathematik und ihrer Grenzgebiete, vol. 4, Chelsea, New York, 1948.

\section{Marshall Hall}

1. Group rings and extensions, I, Ann. of Math. vol. 39 (1938) pp. 220-234.

SAMUEl EIlenberg

1. Topological methods in abstract algebra. Cohomology theory of groups, Bull. Amer. Math. Soc. vol. 55 (1949) pp. 3-37.

Samuel Eilenberg and Saunders MacLane

1. Cohomology theory in abstract groups, I, Ann. of Math. vol. 48 (1947) pp. 51-78.

2. Cohomology theory in abstract groups, II. Group extensions with a non-abelian kernel, Ann. of Math. vol. 48 (1947) pp. 326-341.

3. Algebraic cohomology groups and loops, Duke Math. J. vol. 14 (1947) pp. 435463.

Ruth Moufang

1. Zur Struktur von Alternativkörpern, Math. Ann. vol. 110 (1935) pp. 416-430.

Hans Zassenhaus

1. The theory of groups (trans. by Saul Kravetz), New York, 1949.

UNIVERSITY OF WISCONSIN 